\title{
Philosophiques
}

\section{Retour vers la nature? Questions féministes, sous la direction de K. Genel, J-B. Vuillerod et L. Wezel, Lormont, Le Bord de l'eau, 2019, 240 pages}

\section{Aurélia Peyrical}

Volume 47, numéro 2, automne 2020

URI : https://id.erudit.org/iderudit/1075144ar

DOI : https://doi.org/10.7202/1075144ar

Aller au sommaire du numéro

Éditeur(s)

Société de philosophie du Québec

ISSN

0316-2923 (imprimé)

1492-1391 (numérique)

Découvrir la revue

Citer ce compte rendu

Peyrical, A. (2020). Compte rendu de [Retour vers la nature ? Questions

féministes, sous la direction de K. Genel, J-B. Vuillerod et L. Wezel, Lormont, Le Bord de l'eau, 2019, 240 pages]. Philosophiques, 47(2), 529-534.

https://doi.org/10.7202/1075144ar d'utilisation que vous pouvez consulter en ligne.

https://apropos.erudit.org/fr/usagers/politique-dutilisation/ 
concrète. La deuxième piste éthique est nommée par P. Guenancia « De la sollicitude à la similitude»(p. 210), elle passe par ce que l'autre souffrant m'apprend dans l'empathie que j'éprouve pour lui sur le mode de la sollicitude. Dans cette rencontre nous apprenons que nous appartenons à la même réalité où alternent épreuves, défis, activités, capacités, aussi bien pour nous que pour tout autre.

P. Guenancia conclut son ouvrage en insistant sur la multiplicité des voies de la conscience qui s'exprime au travers de ces quatre auteurs de premier plan. Toutes ces tentatives d'élucidations des rapports de l'homme au monde, aux autres et à soi s'opposent, de façon différente, à une forme d'objectivisme. Elles assument par contre tacitement ou explicitement le commencement husserlien qui stipule que « toute conscience est conscience de quelque chose».

Le livre de P. Guenancia constitue une excellente introduction à la phénoménologie qui permettra au débutant de se familiariser avec les concepts centraux de cette discipline philosophique, mais il pourra aussi servir à un public plus spécialisé qui saura apprécier l'aspect synthétique de l'ouvrage et les comparaisons très pertinentes que fait l'auteur avec d'autres traditions philosophiques comme le cartésianisme, l'empirisme, ou encore le bergsonisme. La clarté et la rigueur des analyses de P. Guenancia font de son ouvrage un véritable guide pour qui voudrait comprendre comment, à partir des intuitions de Husserl, se sont déployées parfois de façon appositive et critique les philosophies de Sartre, Merleau-Ponty et Ricœur.

OLIVIER CONTENSOU

Université Laval

\section{Retour vers la nature? Questions féministes, sous la direction de K. Genel, J-B. Vuillerod et L. Wezel, Lormont, Le Bord de l'eau, 2019, 240 pages.}

Bien qu'ils s'adressent d'abord à des spécialistes, ces actes de colloque «Féminismes, natures, dominations ${ }^{1}{ }^{~}$ forment une introduction à un enjeu décisif pour le féminisme et pour la philosophie politique: l'utilisation d'arguments naturalistes de toutes sortes. Les textes rassemblés ici présentent des analyses féministes variées de conceptualisations plurielles de la nature, au prisme des diverses formes de domination et d'exploitation des femmes.

L'ouvrage trouve son unité dans une double référence. D'une part à l'héritage de la Théorie critique et des pensées anti-essentialistes (néo-) marxistes. D'autre part aux thèses de l'écoféminisme qu'a fait connaître en

1. Organisé les 18 et 19 octobre 2018 à l'Université de Paris I et de Paris-Nanterre. Seule la contribution de Salima Naït Ahmed «De la "dénaturalisation" à la "renaturalisation" des femmes chez Adorno" a été publiée ailleurs, dans Trajectoires, n 12, 2019. 
France le recueil Reclaim ${ }^{2}$ - ce dernier ayant bénéficié d'une récente médiatisation à l'occasion des Marches pour le climat et des discours autour de la COP 21. Il propose à ce titre une «renaturalisation» de certains combats politiques féministes à partir de la construction d'une analogie, voire d'une équivalence, entre l'oppression-exploitation de la nature et celle des femmes.

L'ouvrage comporte quatre parties. Dans la première, nous trouvons trois articles qui portent sur les enjeux de la déconstruction du concept de nature. Le premier, écrit par A. Benoit, est consacré à Butler et présente sa théorie constructiviste de l'identité sexuée ainsi que la conception du langage qui la soutient. Si le dialogue que l'autrice établit entre Butler, Bourdieu et Althusser n'est pas tout à fait nouveau, il permet de saisir l'originalité de Butler, philosophe de la "production discursive de rapports sociaux» (p. 13) qui ne préexistent pas au langage. L'autrice explique en détail comment Butler dépasse Bourdieu dans la théorisation de la matérialité de l'ordre économique, social et politique, mais aussi dans la pensée des pratiques qui le subvertissent en surgissant « depuis le langage lui-même» (p. 21). L'article n'évoque cependant pas le fait que la théorie de Butler octroie un pouvoir peut-être excessif au langage, au détriment d'autres formes de constitution du soi et de subversion des normes. L'article de M. Gérardin-Lavergne répond à cet écueil en expliquant comment le «totem» (notamment féministe) de la "déconstruction " peut mener à une attitude désinvolte vis-à-vis de la force des normes sociales. Elle propose de cesser de fétichiser la dénaturalisation, et, avec l'écoféminisme, de produire un "contre-paradigme au dualisme: un concept de "différence non hiérarchique" » (p. 53). La démonstration de T. Crespo va dans le même sens puisqu'il explore, grâce à des références précises et lumineuses, la façon dont la science est traversée par le pouvoir d'une façon qui peut à la fois justifier l'attitude essentialiste, et entraîner des erreurs scientifiques. On peut par exemple évoquer les effets de la récente obligation, pour toute étude scientifique, de faire du sexe une variable des études médicales. Dans au moins cinq pour cent des cas, cette mesure amène à exagérer l'importance de la variable "sexe " au détriment d'autres variables - comme le nombre de plaquettes - scientifiquement plus pertinentes (p. 34).

La seconde partie porte sur "la dialectique de la raison à l'épreuve du féminisme». Elle est consacrée à l'évaluation critique de l'apport théorique d'Adorno et de Horkheimer pour un féminisme non essentialiste. L. Wezel et P. Guilibert montrent qu'on trouve chez ces derniers des outils théoriques importants pour critiquer l'exploitation des femmes, mais aussi des positions naturalistes, voire misogynes ${ }^{3}$. Dans l'intéressante reconstitution par

2. E. Hache (dir.), Paris, Cambourakis, 2016.

3. Max Horkheimer écrit dans Notes critiques. Sur le temps présent (1949-1969), Paris, Payot, 1993, p. 32: «D’abord, pourquoi je n'aime pas la mode des slacks: la femme déambule maintenant comme un homme, cigarette aux lèvres, commissures tombantes, front plissé, tout 
L. Wezel de la conception adornienne de la féminité comme «cicatrice», on s'étonne néanmoins d'une décontextualisation, par endroits, de l'intrication du naturel et du culturel. D'autres textes sur les femmes auraient pu être mobilisés, par exemple dans les Minima Moralia ${ }^{4}$, pour situer les réflexions sur la «nature féminine» ou le «caractère féminin ». Une telle contextualisation rend manifeste le décrochage de la Théorie critique vis-à-vis des féminismes réels: ils ne citent par exemple jamais V. Woolf, O. de Gouges, G. Sand. L'article esquisse cependant une discussion originale avec les luttes écoféministes, à partir de l'analyse de la "dichotomie empoisonnée» (p. 75) de la nature et de l'histoire chez Benjamin et Adorno. Cette analyse permet entre autres d'argumenter en faveur de l'entreprise d'une autre écriture de l'histoirE du point de vue des femmes ("Herstory»).

L'article de P. Guilibert compare les stratégies de critique de l'exploitation de la nature et des femmes dans The Death of Nature (1980) de C. Merchant, et dans la Dialectique de la raison d'Adorno et de Horkheimer. Son travail donne un juste aperçu des thèses de l'historienne et philosophe C. Merchant. Historienne de la révolution scientifique des $\mathrm{XVI}^{\mathrm{e}}$ et $\mathrm{XVII}^{\mathrm{e}}$ siècles en Europe, elle montre dans son ouvrage que le passage d'une conception organiciste à une conception mécaniste de la nature a impliqué un renouvellement de l'exploitation des femmes. L'auteur se montre moins mesuré, en revanche, quand il prétend que pour les Francfortois la domination de la nature est "le résultat d'une tendance inhérente et immanente à la rationalité, actualisée par des formations sociales marchandes» (p. 82). Le rapport entre la raison et les formes de l'échange est-il à ce point téléologique chez Adorno et Horkheimer? Autre point problématique, l'interprétation hésitante du rapport des femmes et de la nature selon eux. L'auteur ne rend pas réellement compte du paradoxe qui consiste à affirmer à la fois l'inexistence d'une nature féminine première et la «dénaturalisation» des femmes au cours de l'histoire. Or cette difficulté s'explique aisément si on distingue un usage faible et un usage fort du terme de nature. Les femmes, qui partagent une nature humaine commune (au sens faible, non positif) avec les hommes, ont été naturalisées de force dans l'histoire (cette fois au sens fort de l'attribution positive et limitative de traits "prétendument naturel[s]" qui leur sont assignés en fonction de leur sexe). C'est ce processus qu'Adorno et

\footnotetext{
comme le seigneur de cette civilisation qui foule aux pieds la nature. L'égalité avec l'homme est soulignée, lui dont le rôle civilisateur convient si mal à la femme» (je souligne).

4. Je me permets de renvoyer à un travail (non publié) disponible à: https://www.academia. edu/43945630/Les_femmes_une_constellation_dans_les_Minima_Moralia_de_T_W_Adorno, qui comporte une bibliographie récapitulative (non exhaustive) des travaux consacrés à la conception adornienne de la féminité, à sa position vis-à-vis des transformations sociales concernant les femmes, voire à son «féminisme".
} 
Horkheimer, non sans jeu de mots et en faisant fond sur la dialectique histoire-nature, caractérisent comme une "dénaturation ${ }^{5}$.

La troisième partie, intitulée "Généalogies critiques ", cherche à réévaluer les œuvres de Bachofen et de Hegel pour le compte du féminisme. V. Ciantelli montre que le matriarcat n'équivaut ni à un ordre social sans lois ni à une domination inique des femmes sur les hommes. Au contraire, il permet de penser une justice inconditionnelle sous forme de solidarité envers une "Matrie » potentiellement universelle. Le matriarcat s'oppose à la légalité patriarcale hiérarchisante, dont le principe est la lutte entre des lignages ou les tribus (p. 109). Cette lecture stimulante de Bachofen minimise malgré tout le fait que ce dernier considérait le patriarcat comme une forme non seulement différente mais supérieure d'organisation sociale.

Jean-Baptiste Vuillerod se propose de relever un défi de taille: lire Hegel comme un allié potentiel du féminisme ${ }^{6}$. Il montre de façon convaincante que la nature à laquelle Hegel assigne les femmes est dynamique, et il rappelle à juste titre la critique hégélienne sans détour des sociétés misogynes, comme Rome ou «l'Orient ». Cependant, outre les textes qui dénient aux femmes l'égalité juridique mais aussi toute forme d'individualité, on achoppe sur l'interprétation de la lecture hégélienne du mythe d'Antigone. L'auteur voit une "potentialité critique importante dans la définition hégélienne de la féminité comme éternelle ironie de la communauté »(p. 124). Pourtant l' «ironie » chez Hegel signifie la «contestation sans relève dialectique » (p. 125), autrement dit précisément le type de négation indéterminée qui fait problème pour l'idéalisme absolu. Antigone (ni épouse ni mère) reste irrémédiablement extérieure à l'histoire. Elle n'est nullement envisagée par Hegel comme un(e) potentielle citoyen(ne)-sujet(te), car elle parle depuis ce qui, pour le patriarcat, est un "non-lieu» politique. Alors que pour l'individu mâle l'arrachement à la communauté est condition de possibilité d'un devenir sujet individualisant, pour l'individu femelle qu'est Antigone la remise en question du partage entre dikè, la loi de la cité, et thémis, la coutume de l'ordre établi", conduit directement au suicide. Le "potentiel critique» du «non-identique» incarné par Antigone et ses sœurs de lutte ne

5. La référence à l'allemand, qui distingue «Frau» et "Weib», est nécessaire ici. Si le premier terme ne renvoie à aucune distinction hiérarchique, le second en revanche désigne la «nature " prétendument originaire et inférieure, produite par la domination: "L'homme qui maîtrise refuse à la femme (Frau) l'honneur de s'individualiser. L'unité [femme] est socialement un exemple de son genre. Elle représente son sexe, et en cela, en tant qu'entièrement tombée sous la logique masculine, elle tient lieu de nature, le substrat d'une subsomption sans fin en idée, d'une soumission sans fin en réalité. La femme (Weib), en tant qu'être prétendument naturel est le produit de l'histoire, qui la dénature » in T.W. Adorno, Gesammelte Schriften, Bd. 3, p. 132 (nous traduisons).

6. Les ouvrages féministes critiques à l'égard de Hegel sont d'ailleurs légion. Cf. J-B. Vuillerod, Hegel féministe (Paris, Vrin, 2020, à paraître).

7. F. Duroux, Antigone encore. Les femmes et la loi, Éditions Côté-femmes, Paris, 1993. 
peut dès lors faire écho à la dialectique négative d'Adorno qu'à condition de rompre avec la dialectique réconciliatrice de $\mathrm{Hegel}^{8}$, sans pour autant tomber dans le culte post-moderne de la différence - attitude du reste fort peu adornienne.

La quatrième et dernière partie pose la question du défi, pour la critique, des rapports entre féminisme et naturalisme. Dans un premier article sur Arendt dont elle est spécialiste ${ }^{9}$, Katia Genel se demande s'il est possible de reprendre certains des concepts arendtiens malgré son hostilité vis-à-vis du féminisme. K. Genel n'est pas la première à proposer cette réappropriation critique $^{10}$. A. Cavarero, qu'elle cite ${ }^{11}$, s'inspire par exemple d'Arendt pour conceptualiser le rapport au monde comme une «inclination» (p. 142). En réponse aux éthiques et aux politiques (néo)-kantiennes (libérales) de la droiture abstraite, on pourrait penser avec Arendt l'importance de cette «inclination» comme condition d'un agir raisonnable, au nom de la vulnérabilité des corps et la dépendance relationnelle humaine originaire. Cela n'est cependant possible qu'à condition d'extraire ce concept de la dichotomie arendtienne entre l'œuvre (anté- voire anti-politique) et l'action. Alors que la réception d'Arendt dans les sciences humaines se polarise ${ }^{12}$, les difficultés d'un projet de réappropriation sont de taille. L'ontologie politique d'Arendt, fondée sur l'hypostase de la natalité, un concept indéniablement "ontologique», fait notamment problème ${ }^{13}$. Plus critique, le texte de C. Nouët, résolument marxien, expose les stratégies matérialistes de M. Delphy et R. Scholz pour contrer le naturalisme biologisant du «sexe» qui refait régulièrement surface dans la pensée féminisme. Cette dernière montre que le «féminin» découle d'un processus historique de "désocialisation de la production domestique» (p. 166).

Ce dernier article introduit à la traduction des premières pages d'un ouvrage de Scholz, alors inédit en français, intitulé Das Geschlecht des

8. E. Renault, "Adorno hégélien ou anti-hégélien? ", à paraitre dans les actes du colloque «Dialectique et négativité » (Paris I et Maison Heinrich Heine, 2016).

9. K. Genel Hannah Arendt, l'expérience de la liberté, Paris, Belin, 2016.

10. Voir par exemple: K. Genel, «Prendre la parole et être entendu. Lectures de l'analyse arendtienne de l'espace public par Seyla Benhabib et Iris Marion Young", in Raisons politiques, $n^{\circ} 68,4,2017$, p. 47-63.

11. A. Cavarero, «Hannah Arendt: "A Child Has Been Born unto Us ", in Inclinations. A Critique of Rectitude, Stanford, SUP, 2016.

12. E. Faye, Arendt et Heidegger, extermination nazie et destruction de la pensée, (Paris, Fayard, 2016) et plus récemment E. Faye, Y. Bock (dir.) Hannah Arendt, la révolution et les droits de l’homme (Paris, Kimé, 2019).

13. Dans ses leçons sur la révolution (1966-1967), Arendt écrit: «[P]uisque "agir" a toujours signifié mettre en marche quelque chose qui n'était pas là auparavant, la naissance, la natalité humaine, qui correspond à la mortalité humaine, est la condition ontologique sine qua non de toute politique ", in La liberté d'être libre, trad. Françoise Bouillot (Payot, 2019), p. 78. La natalité est le pendant chez Arendt de «l'être-pour-la-mort» heideggérien. 
Kapitalismus $^{14}$. Théoricienne centrale de la Wertkritik (critique de la valeur), Scholz cherche à penser le rapport entre patriarcat et capitalisme pour dépasser tant les féminismes du droit abstrait que ceux de l'intersectionnalité. Elle s'oppose aussi à la déconstruction, qui a conduit selon elle à «l'hypostase postmoderne de la différence»(p. 171-172). Elle se réapproprie Marx et Adorno dans un contexte d'effondrement du "socialisme réel ", et situe le féminisme comme un enjeu central de la Wertkritik. La dissociation, dans la production capitaliste des marchandises, entre le féminin (travail domestique) et la valeur (travail abstrait) a selon elle produit des propriétés dites "féminines ", qui ont été en même temps assignées aux femmes. On peut retenir de cette introduction le concept original de «flexi-sujet» ou «sujet contraint-flexible ${ }^{15}$ » (Zwangs-flexi-Subjekt), qui désigne le sujet post-moderne aliéné. Son livre entend montrer comment se rejoue en lui la hiérarchie des sexes, non pas abolie mais transformée en une aliénation multiforme, plurielle et fragmentée.

Cet ouvrage, dont les contributions ont cherché à réviser les coordonnées de problèmes qui continuent de faire débat, tant en philosophie qu'en sciences humaines, s'achève ainsi de façon ouverte. S'ils interpellent tout particulièrement les philosophes féministes, les discours au sujet du « retour vers la nature", qu'ils soient actuels ou traditionnels, concernent aussi la philosophie en général, dès lors qu'elle ne conçoit pas les faits sociaux et leur interprétation comme un détour ou un obstacle. Dans ces circonstances, il n'est plus possible pour elle de faire l'impasse sur les analyses féministes, qu'elles s'inspirent ou non, comme ici, du matérialisme et des pensées critiques.

AURÉLIA PEYRICAL

Université Paris-Nanterre

\section{Olivier Sartenaer, Qu'est-ce que l'émergence?, Paris, Vrin, 2018, 128 pages.}

L'attrait philosophique de l'émergence, estime Sartenaer, tient à la position mitoyenne et médiatrice que ce concept occupe entre réductionnisme et dualisme, position à partir de laquelle «l'existence de certaines réalités d'ordre supérieur peut être soutenue et légitimée» (p. 12). Mais cet attrait s'accompagne d'une tension inhérente, qu'il résume ainsi: "[C]omment peut-on encore être physicaliste - c'est-à-dire penser que tout est physique, ou que tout peut ultimement s'ancrer dans le monde physique - sans penser du même coup que tout est réductible en principe au monde physique? » (p. 25).

14. R. Scholz, Le sexe du capitalisme. Masculinité et féminité comme piliers du patriarcat producteur de la marchandise, Paris, Crise \& Critique, 2019.

15. Notre traduction. 